\title{
Molecular studies in schizophrenia
}

\section{Estudos moleculares em esquizofrenia}

\section{Daniel Martins-De-SouZa1 ${ }^{1}$ Bruno M. De Oliveira²}

1Max Planck Institute of Psychiatry \& Ludwig Maximilians University (LMU), Munich, Germany; Laboratory of Neurosciences (LIM-27), Institute of Psychiatry, School of Medicine, University of Sao Paulo (IPq-FMUSP), Sao Paulo, SP, Brazil.

2 Laboratory of Proteomics, Department of Biochemistry, State University of Campinas (Unicamp), Campinas, SP, Brazil.

Received: 9/23/2012 - Accepted: 11/7/2012

Martins-de-Souza D, Oliveira BM / Rev Psiq Clín. 2013;40(1):1

Alarming numbers about psychiatric disorders for the next decades have been forecasted by the world health organization (WHO). The estimation is that until 2030 four persons in ten retired from their jobs will do it due to a psychiatric disorder. In addition, whilst rising attention and funding have been given to treatment and research of cardiac diseases, cancers and diabetes, the same does not occur for psychiatric illnesses. On the contrary, in Brazil psychiatric clinics have been closed and health insurance companies have even been classifying psychiatric treatments as non-essential. Biological evolution has not followed social evolution leading to the development of several modern diseases, including psychiatric disorders. Since medicine aims for the improvement of human life, it is important that medical research target both, physical and mental health.

Schizophrenia is not only one of the most intriguing psychiatric disturbances, but probably the most complex one to be studied. This is due to the diverse array of presented symptoms and their overlap with those from different psychiatric disorders. As importantly, the multifactorial characteristic of schizophrenia, which is based in molecular and environmental factors, makes it difficult the establishment of pre-clinical models. Consequently, their validation has been controversial.
The pathobiology of schizophrenia has been largely investigated in the past years. Scientific articles have been published about fundamental knowledge, pre-clinical and clinical aspects and even research involving stem cells, denoting crescent efforts and scientific interest in the field. However, some of the most elementary features of schizophrenia pathogenesis were still not resolved. While basic molecular elements have been well characterized, there is still a lack of data integrating these results. Schizophrenia diagnosis is strictly clinic and prognostic intervention is almost inexistent. Medication effects must be more characterized in order to promote more effective treatment, excluding undesirable side effects as well as providing targeted treatment for each particular patient according to their stratification status. The moment shows us that there is a need to invest more efforts to connect the molecular aspects of schizophrenia.

With this in mind, Revista de Psiquiatria Clínica offers this special edition about "Molecular studies in schizophrenia". This edition is composed by eight articles from research groups from different countries ranging from basic science, passing through preclinical models up to biomarkers identification. Our intention is to deliver knowledge about the newest approaches on schizophrenia and psychiatric research, aiming to generate to our readers new ideas and inspire new projects which may add pieces to the schizophrenia puzzle. 